Unfallchirurg 2013 $\cdot 116: 8-9$

DOI 10.1007/s00113-012-2297-6

Online publiziert: 21. Januar 2013

(c) Springer-Verlag Berlin Heidelberg 2013

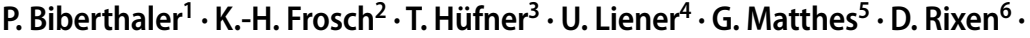 G. Schmidmeier ${ }^{7} \cdot$ C.G. Wölffl ${ }^{8}$
}

${ }^{1}$ Klinik für und Poliklinik für Unfallchirurgie, Klinikum rechts der Isar, Technische Universität München

${ }^{2}$ Chirurgisch-Traumatologische Zentrum, Klinik St. Georg Hamburg

${ }^{3}$ Wirbelsäulenzentrum Hannover

${ }^{4}$ Klinik für Orthopädie und Unfallchirurgie, Marienhospital Stuttgart

${ }^{5}$ Klinik für Unfallchirurgie und Orthopädie, UKB Berlin

${ }^{6}$ Klinik für Unfallchirurgie und Orthopädie, BG-Klinik Duisburg

${ }^{7}$ Sektion Unfallchirurgie, Abteilung Orthopädie und Unfallchirurgie, Universität Heidelberg

${ }^{8}$ Klinik für Unfallchirurgie und Orthopädie, BG-Klinik Ludwigshafen

\section{Das junge Forum der DGOU}

\section{Nachwuchs im Fach Orthopädie und Unfallchirurgie}

schluss, einen neuen gemeinsamen Facharzt für Orthopädie und Unfallchirurgie zu schaffen. Diese Entwicklung führte zu einer erheblichen Unsicherheit bei den Mitarbeitern der unfallchirurgischen Kliniken und beide Freunde wussten über Kollegen zu berichten, die aufgrund dieser Unsicherheit die Fachrichtung nicht mehr als Karriereziel in Betracht zogen. Rasch war man sich einig, dass die Zusammenlegung der beiden Fachärzte enorme Chancen für gerade diese jüngeren Assistenten bieten, jedoch auch eine Reihe von Risiken bergen würde. Im Rahmen der weiteren Diskussion wurde rasch klar, dass in vielen Arbeitsgruppensektionen und Ausschüssen der entsprechenden wissenschaftlichen Vertretungen sowie Expertenrunden junge Ärzte kaum vertreten waren.

Die Diskussion entwickelte sich dann lebhaft mit kreativen Ideen, was nun die besten Maßnahmen sein könnten. Dabei kristallisierte sich mehr und mehr heraus, dass der unfallchirurgische Nachwuchs keine adäquate Interessensvertretung hätte. Aus dieser Initiative heraus wurde eine Reihe von engagierten jungen Fachärzten angesprochen, ob sie nicht Interesse hätten mitzumachen. So stießen die oben genanten Gründungsmitglieder hinzu und man war sich zügig darüber ei- nig, dass diese Interessensvertretung nicht nur die oben angesprochenen berufspolitischen Ziele im Auge haben, sondern gerade auch eine wissenschaftlich fundierte Ausrichtung als wesentliches Ziel vertreten sollte ( $\bullet$ Abb. 1). Daraufhin folgte ein umfangreiches Brainstorming, wie man so ein Projekt am ehesten realisieren und zum Erfolg führen könnte. Hierfür bot sich die Deutsche Gesellschaft für Unfallchirurgie (DGU) als wissenschaftliche Fachgesellschaft und damit auch Interessensvertretung der Unfallchirurgie in Deutschland an. Da alle Gründungsmitglieder langjährige Mitglieder derselben waren, entwickelten sie eine Strategie, wie so eine Interessensvertretung innerhalb der DGU initialisiert werden könnte. Somit wurden die Ideen dann mit wesentlichen Entscheidungsträgern der DGU, dem Generalsekretär und dem Vorstand diskutiert und nach deren sehr positiven Rückmeldung und Unterstützung eine Gründungssitzung der besagten Interessensvertretung einberufen. Dort wurde unter dem Namen „Junges Forum“ der Grundstein für ein Sprachrohr des unfallchirurgischen Nachwuchses gelegt. Ziel der Gruppierung war es, junge Fachärzte und Oberärzte zur Mitarbeit zu bewegen und anhand von klar strukturierten Aufgaben die Interessen des unfallchirurgi- 

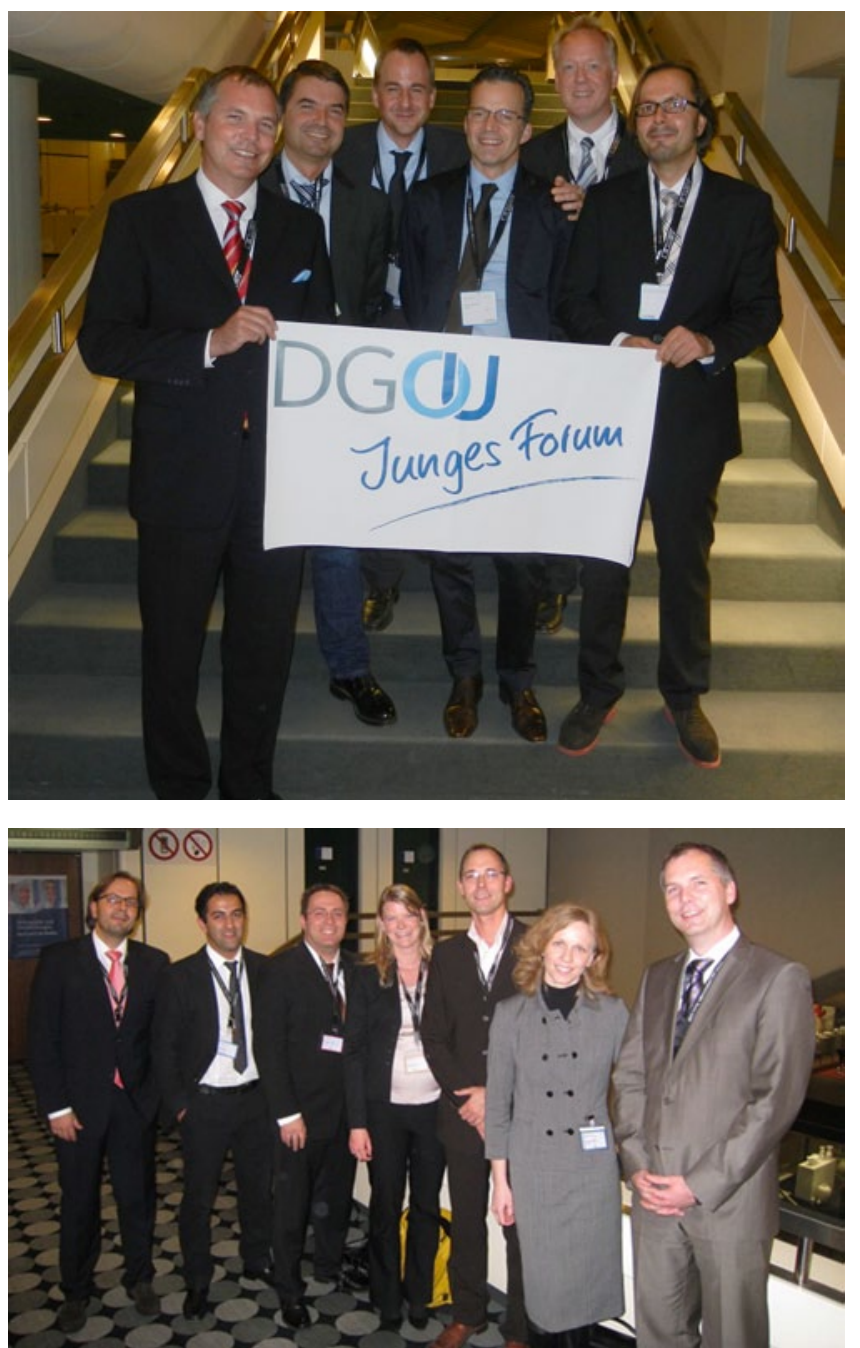

schen Nachwuchses zu vertreten. Erste Projekte waren eine Job-Börse, Bearbeitung neuer Arbeitszeitmodelle, eine Wissenschaftsbörse, Hospitationen und eine Gruppe, die sich um Weiterbildung gekümmert hat.

Im Jahre 2006 konnten Gerrit Matthes und Christoph Wölfl die Leitung des Jungen Forums fortsetzen. Hierunter erfolgte die intensive Kontaktaufnahme zu Kollegen der orthopädischen Fachgesellschaft DGOOC, so dass entsprechend der $\mathrm{Zu}$ sammenlegung des Faches auch ein gemeinsames junges Forum der Deutschen Gesellschaft für Orthopädie und Unfallchirurgie gegründet werden konnte. 2009, 6 Jahre nach der Initialzündung, konnten Gerrit Matthes und Christoph Wölfl die Erfolgsstory an die nächste Generation übergeben (• Abb. 2). Mittlerweile konnten Nachwuchsförderprogramme wie die „Summer School“, welche als Idee aus dem Jungen Forum entstand und der Tag der
Abb. $2<$ Übergabe an das neue Team

Abb. $1<$ Das Junge Forum der DGOU; Gründungsmitglieder
Studierenden auf der Jahrestagung in Berlin, etabliert werden. Gremienarbeit und Umfragen über Ausbildungssituation und Weiterbildung runden die Tätigkeit des Jungen Forums ab. Wir denken, dass das Junge Forum ein maßgeblicher Faktor für eine junge, moderne, zukunftsorientierte Fachgesellschaft ist. Hier gilt unser Dank allen uns nachfolgenden Akteuren und Mitstreitern und im Besonderen auch den Förderern innerhalb der Fachgesellschaft. Ganz besonders bedanken möchten wir uns bei all jenen Kollegen, die unser Projekt mit viel Herzblut mitgetragen und andere in ihrem Umfeld zur Mitarbeit motiviert haben.

Vorbild ist das Junge Forum auf jeden Fall geworden. Bis heute hat keine andere chirurgische Fachgesellschaft etwas Vergleichbares zu bieten. Es freut uns sehr, dass das Präsidium der DGCH nun auch eine Initiative des Jungen Forums der DGOU unterstützt, die Arbeit und Be- mühungen der Jungen Foren aller chirurgischen Fachgesellschaften zu bündeln. Dazu organisiert das Junge Forum der DGOU nun erstmals eine Perspektivtagung Chirurgie in München, um so junge Vertreter aller Fachdisziplinen an einen Tisch zu bekommen.

\section{\) Das Fachgebiet Orthopädie und Unfallchirurgie ist zu einem der interessantesten Fachgebiete in der Weiter- bildungslandschaft geworden}

Wir freuen uns auf die Zukunft! Da wir weitgehend in verantwortlichen Positionen tätig sind hoffen wir auf die zukünftige Zusammenarbeit mit engagierten Nachwuchsärzten innerhalb des jungen Forums und werden das Junge Forum weiterhin unterstützen. Das Fachgebiet Orthopädie und Unfallchirurgie ist durch die Zusammenlegung zu einem der spannendsten und interessantesten Fachgebiete in der Weiterbildungslandschaft geworden und wir können nur allen jüngeren Kollegen empfehlen, ihr Ziel konsequent zu verfolgen. Die Tätigkeit in diesem Gebiet ist eine verantwortungsvolle aber auch erfüllende Aufgabe, daher würden wir uns sehr freuen, wenn sich alle jungen Kollegen angesprochen fühlen beim Jungen Forum mitzumachen und ebenfalls zu versuchen, die Bedingungen ihres eigenen persönlichen Arbeitslebens positiv mit zu gestalten.

Herzlichen Glückwunsch zu diesem Themenheft.

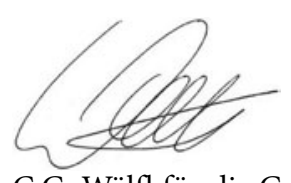

C.G. Wölfl für die Gründungsmitglieder des Jungen Forums

\section{Korrespondenzadresse}

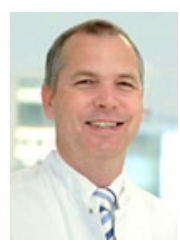

\section{C.G. Wölfl}

Klinik für Unfallchirurgie und Orthopädie, BG-Klinik Ludwigshafen Ludwig-Guttmann-Straße 13, 67071 Ludwigshafen christoph_woelfl@hotmail.com 